\title{
Recurrent discitis in the acute rehab setting: a case report
}

\author{
Kunj Patel ${ }^{1} \cdot$ Lauren Hutts $^{2} \cdot$ Stephen Porter ${ }^{1} \cdot$ Zachary Fausel $^{1} \cdot$ Dale Strasser $^{1}$
}

Received: 29 October 2017 / Accepted: 9 February 2018

(c) International Spinal Cord Society 2018

\begin{abstract}
Introduction Discitis is an infection of the intervertebral disc space that can lead to paralysis, sepsis, epidural abscess, or other life-threatening complications if left untreated and may sometimes present with limited laboratory abnormalities to clue a diagnosis.

Case presentation We present a case of a 67-year-old female in an acute rehabilitation setting who had presenting symptoms of sudden unexplained back pain refractory to pain control. She had a previous history of discitis and multiple failed knee replacement surgeries secondary to MSSA infections. Although vitals and basic labs were within normal limits, we recognized the clinical presentation as an indicator to order inflammatory markers (CPR and ESR), which were elevated compared to levels on admission, and subsequently ordered imaging and blood cultures to detect a case of recurrent worsening discitis. Discussion We highlight the presentation of recurrent discitis and necessary low threshold needed to detect such recurrence in patients with a previous history of discitis that have sudden unexplained back pain with no other usual physical or laboratory indicators of infection.
\end{abstract}

\section{Introduction}

Discitis is an infection of the intervertebral disc space that can lead to paralysis, sepsis, epidural abscess, or other lifethreatening complications if left untreated [1-4]. Incidence occurs as frequently as 2.4 persons in every 100,000 [5]. The lumbar region is most frequently affected (50\%), followed by the thoracic region $(33 \%)$, and then the cervical region (3-10\%) [6]. It has a bimodally distributed age of presentation, occurring most commonly in children (average age of 7 years) and adults in the sixth and seventh decades of life, with a 2:1 male predominance in adults [1, $3,5]$. If left untreated, discitis has an associated $2-12 \%$ mortality $[1,3]$.

It occurs most commonly by hematogenous seeding, usually from urinary tract infections that spread to the spinal epidural space via Batson's plexus, but also can occur in the postoperative setting in approximately $1-2 \%$ of patients

Stephen Porter

stport03@gmail.com

1 Department of Rehabilitation Medicine, Emory University School of Medicine, Atlanta, USA

2 Department of Physical Medicine and Rehabilitation, University of Alabama-Birmingham School of Medicine, Birmingham, USA undergoing spinal surgeries $[1,6]$. Some of the most common infectious agents responsible for discitis are the same agents that cause urinary tract infections, mainly Staphylococcus aureus (32-67\%) and Escherichia coli (21\%); though Pseudomonas species $(6 \%)$ are also common and should be suspected in IV drug users [1, 3-5, 7]. In the setting of sudden onset back pain in a high risk patient, elevated erythrocyte sedimentation rate and C-reactive protein may be the only abnormal laboratory values to help indicate suspicion for recurring discitis $[4,7]$. Prompt treatment with appropriate antibiotics following culture of organism, and stabilization of the spine for prevention of damage during recovery is then recommended for the best outcome of the patient [7]. Having a low threshold for ordering inflammatory markers in the presence of sudden onset unexplained back pain in a high risk patient is crucial for the early detection of recurrent discitis. In this paper, we present a case of recurrent discitis in a high risk patient that had a previous history of discitis whose only clinical indicators were sudden unexplained back pain and inability to tolerate therapy sessions.

\section{Case presentation}

A 67-year-old female was admitted to the acute rehabilitation hospital for T4 Asia B spinal cord injury (SCI) 
secondary to spinal cord infarction. Prior to her SCI, she had T5-6 discitis due to methicillin sensitive staphylococcus aureus (MSSA), and a history of four failed left knee replacement surgeries, complicated by MSSA infection. She had completed a 16 week course of IV antibiotics prior to her arrival, and while at rehab initially demonstrated excellent participation in physical therapy and decreasing requirement of pain medications. However, after the first week, she suddenly began experiencing increasing back pain. She did not appear acutely ill, and her vitals and labs were unremarkable. Over the next couple days, her pain progressed despite aggressive medication adjustments, and she failed to tolerate physical therapy. A CRP and ESR were ordered, which returned $>380$ and 76 , respectively-a significant increase from preadmission levels of 11 and 37 , respectively. IV antibiotics were restarted, and blood cultures were obtained. Repeat imaging (Fig. 1) showed worsening discitis T3-T6 with 2 right paraspinal muscular abscesses and significant circumferential epidural phlegmon T2-T7. Blood cultures grew MSSA.

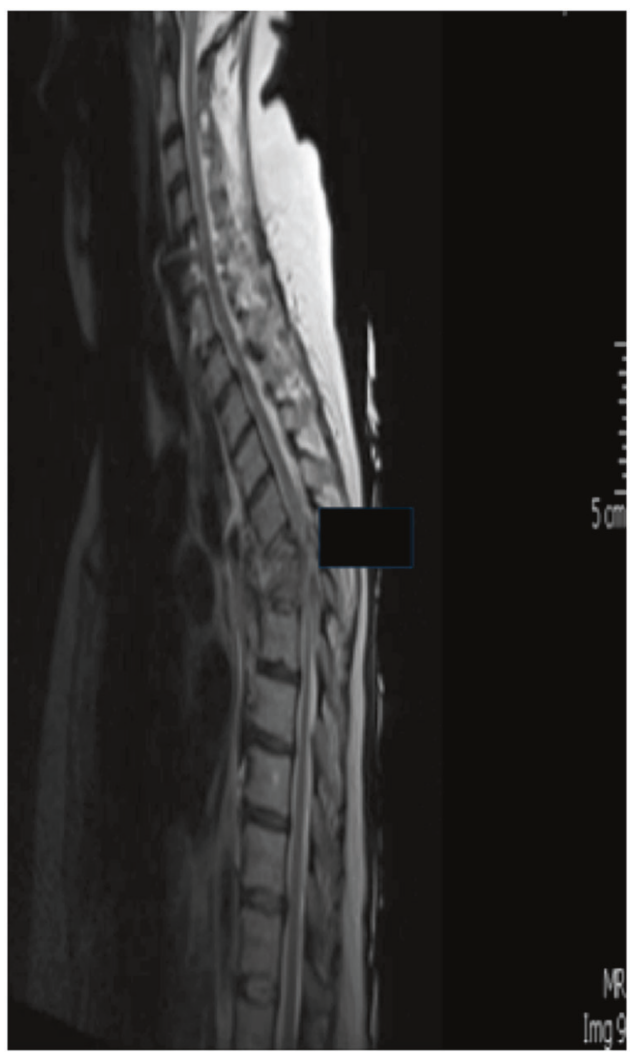

Fig. 1 T2-weighted MRI c-spine after readmission to acute care hospital demonstrating advanced discitis with accompanying osteomyelitis at T4 and T5 with circumferential epidural phlegmon

\section{Discussion}

Discitis is a serious and rare condition which can cause significant neurological injury. Discitis is thought to spread to the involved intervertebral disc via hematogenous spread of a systemic infection either through the metaphyseal arteries or Batson's plexus, with urinary tract infections, pneumonia, and soft-tissue infection seeming to be the most common sources [3]. Often, no other site of infection is discovered [3, 4]. Frequently, discitis presents without any systemic signs, leukocytosis, or superficial signs of infection $[3,5]$. Back pain and ESR/CRP elevation are the most consistent abnormalities seen in cases of discitis [3, 4, 7, 8]. Blood cultures are positive in only one third to one half of cases, and recurrence of infection occurs in $2-8 \%$ of patients [3, 9].

\section{Clinical presentation and diagnosis}

The discitis patient usually presents with sudden worsening back pain and spasm of the paraspinous musculature in the general region affected, sometimes with localized tenderness and/or pyrexia [1, 3, 4]. As the infection spreads to nearby structures such as involvement of more discs, adjacent vertebral bodies, and compresses neural structures, the pain becomes more intense. Medical histories that should increase clinical suspicion include comorbidities such as diabetes (most common), history of spinal trauma or fracture, AIDS or history of IV drug use, steroid use, cancer, cirrhosis, chronic kidney disease, or presence of other known foci of infection [3-5, 9]. Early stages can also present as vague constitutional symptoms such as low-grade fever, anorexia, or malaise, but can eventually progress to frank sepsis with high-grade fever, rigors, and chills [1]. With administration of steroids, the patient's presentation in symptoms may be delayed due to the masking of early clinical indicators [1]. In later stages of infection, neurologic findings such as sensory or motor deficit, or bowel and bladder symptoms may be seen due to the resultant spinal nerve root or spinal cord compression [1]. The most common physical sign is localized tenderness over the involved area with concurrent paraspinal muscle spasm [3]. If untreated, resultant spread into the nearby epidural space can compromise the vascular supply with resultant ischemia, spinal cord infarction, and permanent neurological deficits, as seen in our patient's case [1].

In the presence of clinical suspicion, current guidelines recommend the following: complete neurologic examination, laboratory evaluation (complete blood count, erythrocyte sedimentation rate, C-reactive protein, basic 
metabolic panel, urinalysis, urine culture, and 2 sets of blood cultures), stat imaging of the spine with and without contrast, ideally within $2 \mathrm{~h}$ of abnormal neurologic findings or within $6 \mathrm{~h}$ of normal neurologic findings, and biopsy within $24 \mathrm{~h}$ if there is evidence of discitis on imaging but blood cultures are negative $[1,5]$. Antibiotics should then be started while workup is pending. Plain radiographs typically show evidence of disc space narrowing and endplate changes suggestive of discitis, though may not be apparent until weeks after onset of the disease [1,3]. CT scans can detect discitis earlier than plain radiographs, but MRI is the most sensitive and specific imaging in the detection of discitis [3].

\section{Treatment}

Rapid treatment of discitis aims to achieve two goals: 1) Use antibiotics to treat the infection, and 2) drainage of any abscess formation to relieve compression on nearby neural structures [1]. Since Staphylococcus aureus is the most common organism involved, antibiotics such as vancomycin should be started immediately after blood and urine samples are taken, with adjustments to therapy regimen made as more information becomes available through culture reports [5]. Other organisms such as Mycobacterium tuberculosis or fungi should be considered in immunocompromised patients or cases that do not respond to typical antibiotic regimens. Parenteral treatment for 6-8 weeks should be given but should not be discontinued until ESR has dropped by one half to one third, and the patient should not have pain on ambulation or signs of neurologic deficits before discontinuing regimen [3]. Bed rest for 2 weeks followed by external bracing and immobilization of spine for 3-6 months when moving is indicated to help stabilize affected spinal segments [3]. This should help improve long-term outcome of patients suffering from discitis in order to prevent collapse of the vertebral segments and kyphos formation [1,3].

\section{Conclusion}

A typical presentation for recurrent discitis is sudden worsening back pain in a high risk patient in the absence of other usual physical or laboratory signs of infection. As a result, clinicians should have a low threshold to test for inflammatory markers (ESR, CRP) in these patients when any clinical suspicion is present. Follow up with the collection of other appropriate labs (UA, urine, and $2 \times$ blood cultures), stat imaging, and biopsy, if indicated, so that appropriate antibiotics and therapy regimen can be administered swiftly.

\section{Compliance with ethical standards}

Conflict of interest The authors declare that they have no conflict of interest.

\section{References}

1. Waldman S. Diskitis. Atlas of common pain syndromes. 3rd edn. Philadelphia: Saunders; 2008. p. 252-5.

2. Goel V, Young J, Patterson C. Infective discitis as an uncommon but important cause of back pain in older people. Age Aging. 2000;29:454-56.

3. Marcovici A, Goldstein J. Diskitis. Emedicine. http://emedicine. medscape.com/article/1263845-overview Accessed 1 Sep 2015.

4. McCausland E, Liggett N, Forbes R. Infective discitis in a district general hospital. Ulst Med J. 2008;77:138.

5. Chenoweth C, Bassin B, Hartley S, Mack M, Kunapuli A, Park P, et al. Vertebral osteomyelitis, discitis, and spinal epidural abscess in adults. Quality Management Program of University of Michigan 2013;1-11. http://www.med.umich.edu/1info/FHP/practiceguides/ vertebral/VO.pdf Accessed 1 Sep 2015.

6. Go J, Rothman S, Prosper A, Silbergleit R, Lerner A. Spine infections. Neuroimaging Clin N Am. 2012;22:755-72.

7. Silber J, Anderson D, Vaccaro A, Anderson P, McCormick P. Management of postprocedural discitis. Spine J. 2002;2:279-87.

8. Honan M, White G, Eisenberg G. Spontaneous infectious discitis in adults. Am J Med. 1996;100:85-9.

9. Parra J, Martin A, Martinez-Aedo A, Ivanez I, Agreda E, Stern L. Management of infectious discitis. Outcome in one hundred and eight patients in a University Hospital. Int Orthop. 2012;36:239-44. 